\title{
Maternal depressive symptoms during and after pregnancy and child developmental milestones
}

Soile Tuovinen ${ }^{1 *}$, Marius Lahti-Pulkkinen ${ }^{1-3^{*}}$, Polina Girchenko ${ }^{1}$, Jari Lipsanen ${ }^{1}$, Jari Lahti ${ }^{1,4}$, Kati Heinonen $^{1}$, Rebecca M Reynolds ${ }^{2}$, Esa Hämäläinen ${ }^{5}$, Eero Kajantie ${ }^{3,6,7}$, Hannele Laivuori ${ }^{8,9}$, Anu-Katriina Pesonen ${ }^{1}$, Pia M Villa $^{8}$, Katri Räikkönen ${ }^{1}$

\section{Affiliations:}

${ }^{1}$ Department of Psychology and Logopedics, Faculty of Medicine, University of Helsinki, Helsinki, Finland

${ }^{2}$ University/British Heart Foundation Centre for Cardiovascular Science, Queen's Medical Research Institute, University of Edinburgh, UK

${ }^{3}$ National Institute for Health and Welfare, Helsinki, Finland

${ }^{4}$ Helsinki Collegium for Advanced Studies, University of Helsinki, Helsinki, Finland

${ }^{5}$ Department of Clinical Chemistry, Helsinki University Hospital and University of Helsinki, Finland

${ }^{6}$ Children's Hospital, Helsinki University Hospital and University of Helsinki, Helsinki, Finland

${ }^{7}$ PEDEGO Research Unit, MRC Oulu, Oulu University Hospital and University of Oulu, Oulu, Finland

${ }^{8}$ Medical and Clinical Genetics, University of Helsinki and Helsinki University Hospital, Finland

${ }^{9}$ Institute for Molecular Medicine Finland/HiLIFE, University of Helsinki, Finland

*Doctors Tuovinen and Lahti-Pulkkinen had equal contribution to the manuscript and are the joined first authors.

Abbreviated title: Maternal depression \& child neurodevelopment

Keywords: depression, epidemiology, pregnancy and postpartum, maternal-child, cognition.

Conflict of Interest: The authors declare no conflict of interest.

\section{Correspondence to:}

Marius Lahti-Pulkkinen, Ph.D. Address: Department of Psychology and Logopedics, Faculty of Medicine, Haartmaninkatu 3, 00014 University of Helsinki, Finland

Telephone: +358503440662. Email:marius.lahti-pulkkinen@helsinki.fi 
Corresponding Author: Marius Lahti-Pulkkinen. 2

\section{ABSTRACT}

Background: Maternal depressive symptoms during and after pregnancy predict poorer child neurodevelopment. The effects of timing, symptom severity and additive influences remain unclear. Methods: 2231 mothers of the Prediction and Prevention of Pre-eclampsia and Intrauterine Growth Restriction (PREDO) study completed the Center of Epidemiological Studies Depression Scale biweekly up to 14 times during pregnancy and twice up to 12 months after pregnancy. At child's age 1.9-5.7 years, the mothers completed the Beck Depression Inventory-II on their concurrent depressive symptoms and Ages and Stages Questionnaire on child developmental milestones.

Results: Higher mean maternal depressive symptoms, each biweekly score and consistently clinically relevant symptomatology during pregnancy predicted lower total developmental milestones, fine and gross motor, communication, problem solving and personal/social skills scores in children. While maternal depressive symptoms up to 12 months after pregnancy and in early childhood also predicted lower developmental milestones scores, developmental milestones scores were the lowest in children whose mothers' depressive symptoms were above the clinical cutoff either only during pregnancy, both during and up to 12 months after pregnancy, or at each three time-points.

Conclusion: Maternal depressive symptoms during pregnancy, in the first year postpartum and in early childhood are associated with poorer child neurodevelopment. Our findings further suggest that antenatal and post-pregnancy depression have additive effects on neurodevelopment. Children of mothers with the most chronic and severe depressive symptoms during pregnancy had the most neurodevelopmental disadvantages. Our findings emphasize the adverse effects of maternal depression during and after pregnancy and in early childhood on child neurodevelopment. 


\section{INTRODUCTION}

Approximately $20 \%$ of women experience clinically relevant depressive symptoms during pregnancy(Lahti et al., 2017; Molyneaux, Poston, Ashurst-williams, \& Howard, 2014). In over $40 \%$ of them, the symptoms continue postpartum(Heron, O'Connor G., Evans, Golding, \& Glover, 2004), and 80\% of women with postpartum depression have subsequent depressive episodes(Halligan, Murray, Martins, \& Cooper, 2007). Depressive symptoms not only complicate maternal health during and after pregnancy but also predict low birth weight(Jarde et al., 2016), preterm birth(Grigoriadis et al., 2013; Jarde et al., 2016; Pesonen et al., 2016), caesarean section delivery(Chung, Lau, Yip, Chiu, \& Lee, 2001), decreased breastfeeding rates(Grigoriadis et al., 2013), and suboptimal mother-child attachment development(Misri \& Kendrick, 2008).

According to the Developmental Origins of Health and Disease (DOHaD)-hypothesis(Barker, 2007), maternal depression during and after pregnancy may also affect child neurodevelopment through developmental programming, permanently changing the structure and functioning of organs and body's biological feedback systems, thereby compromising child neurodevelopment. Accordingly, maternal depression during pregnancy has been associated with structural brain changes in child prefrontal cortex(Sandman, Buss, Head, \& Davis, 2015) frontal and inferior temporal areas(Lebel et al., 2016), and amygdala(Rifkin-Graboi et al., 2013), and in neural connectivity between amygdala and fronto-striatal areas(Qiu et al., 2015; Scheinost et al., 2017; Soe et al., 2017). Maternal depression postpartum has been associated with child cortical thickness and white matter diffusivity in right superior frontal areas (Lebel et al., 2016).

Furthermore, several studies have shown that maternal depressive symptoms during and after pregnancy may be associated with poorer neurodevelopmental outcomes in the offspring(Carter, Garrity-Rokous, Chazan-Cohen, Little, \& Briggs-Gowan, 2001; El Marroun et al., 2016; Evans et al., 2012; Gerardin et al., 2011; Grace, Evindar, \& Stewart, 2003; Hay, Pawlby, Waters, \& Sharp, 2008; Y. Lin et al., 2017; Räikkönen et al., 2015; Schechter et al., 2016; van der Waerden et al., 2017; Weikum, Oberlander, 
Corresponding Author: Marius Lahti-Pulkkinen. 4

Hensch, \& Werker, 2012). In one of the largest such studies, clinically relevant maternal depressive symptoms at $18^{\text {th }}$ and $32^{\text {nd }}$ gestational weeks associated with lower intelligence quotient (IQ) in 8-year-old children(Evans et al., 2012), while maternal depression at 8 weeks to 8 months or at 21 to 33 months after pregnancy did not predict child IQ. Children whose mothers reported clinically relevant depressive symptoms across all these measurement points had the lowest IQ. In another large study, maternal depressive symptoms at gestation week 20 was associated with child global executive functioning and shifting problems at age 4 years but not with non-verbal cognition at age 5 years or neuropsychological functioning at 7 years(El Marroun et al., 2017). The associations were independent of maternal depressive symptoms 2 months or 3 years after childbirth. However, independent effects of maternal depressive symptoms after childbirth or additive effects of maternal depressive symptoms across timepoints were not examined. Yet, in another large study(van der Waerden et al., 2017), consistently high maternal depressive symptoms from $24^{\text {th }}-26^{\text {th }}$ gestational weeks up to 5 years after childbirth were independently associated with lower IQ scores in 6-year-old children. In contrast, when adjusting for maternal antenatal anxiety, depressive symptoms concurrent to measuring child IQ and perinatal and sociodemographic covariates, child IQ was not associated with maternal depressive symptoms which were elevated only during pregnancy or only after childbirth. Furthermore, some smaller studies have reported null associations between maternal depressive symptoms during(Hay et al., 2008; Ibanez et al., 2015; Nulman et al., 2012) or after(Kurstjens \& Wolke, 2001) pregnancy with child neurodevelopment or associations with even better outcomes(DiPietro, Novak, Costigan, Atella, \& Reusing, 2006; Plamondon et al., 2015).

No previous studies have included measurements of depressive symptoms throughout pregnancy. Hence, it remains unknown if there is a specific neurodevelopmental vulnerability window for maternal antenatal depressive symptoms, or whether consistently elevated depressive symptoms during pregnancy exert the most detrimental effects. As only two studies measured depressive symptoms during and after pregnancy and up to child's preschool age(Evans et al., 2012; van der Waerden et al., 2017), it also remains unclear if maternal depressive symptoms during pregnancy, postpartum and early childhood periods exert different, independent or additive effects on child neurodevelopment. 
Against this background, we tested whether maternal depressive symptoms, measured biweekly throughout pregnancy, twice up to 12 months postpartum, and when the child was 1.9-5.7-yearold, associated with child developmental milestones in early childhood in a large prospective cohort of Finnish mothers and children. Consecutive measurements of depressive symptoms allowed us to address if associations varied by pregnancy stage, by pregnancy, postpartum and early childhood periods, and if consistently elevated levels of depressive symptoms throughout pregnancy, and/or throughout pregnancy to early childhood exerted most detrimental effects.

We hypothesized that maternal depressive symptoms during and after pregnancy and in early childhood would be associated with lower developmental milestone scores in children. We also hypothesized that depressive symptoms during pregnancy would be specifically associated with poorer child neurodevelopment, and that chronically elevated maternal depressive symptoms from pregnancy to early childhood would predict the poorest neurodevelopmental outcomes. The scale we used to measure child developmental milestones, the Ages and Stages Questionnaire (ASQ), measures fine and gross motor, problem solving, communication and personal/social skills. Although findings are inconclusive, maternal depression during pregnancy has been associated with poorer child general problem-solving skills and with poorer psychomotor, social and communicative development(Evans et al., 2012; Koutra et al., 2017; Y. Lin et al., 2017; Weikum et al., 2012). Hence, we hypothesized that maternal depression would be associated with poorer child neurodevelopmental outcomes across all domains. 
Corresponding Author: Marius Lahti-Pulkkinen. 6

\section{METHODS}

Originally, 5332 pregnant women were recruited to the Prediction and Prevention of Pre-eclampsia and Intrauterine Growth Restriction (PREDO)-study when visiting antenatal clinics at study hospitals in Southern and Eastern Finland for first ultrasound screening. Of them, 547 either withdrew participation during pregnancy or had untraceable child's birth dates from Finnish Medical Birth Register (MBR)/hospital records. These women were excluded from the study sample and may include miscarriages and stillbirths not identified due to missing register data. An additional 8 women were excluded since a miscarriage or stillbirth was identified from MBR.

Of the 4777 women who gave birth to a singleton live child in 2006-2010, 3,402(71.2\%) completed the biweekly depressive symptoms questionnaire during pregnancy, and of these 3,312(97.4\%) at either of the two assessments that took place up to 12 months after pregnancy [at $1^{\text {st }}$ assessment at mean=2.41 weeks postpartum;standard deviation $(S D)=1.2$;range:0.1-18.0 weeks postpartum and/or at $2^{\text {nd }}$ assessment at mean=28.3 weeks postpartum;SD=4.2;range:18.4-54.3 weeks postpartum], and $2,667(78.4 \%)$ at the child developmental follow-up at child ages 1.9 to 5.7 years. We have previously reported the study design and follow-up attrition(Girchenko et al., 2017). Of the women with depressive symptoms data during or after pregnancy or at developmental follow-up, 2,231, 2,192 and 2,170, respectively, had child developmental milestones data at child's mean age 3.5(SD=0.7;range:1.9- 5.7) years. Supplemental Table 1 shows comparisons of participants and non-participants.

All participating women signed informed consents. Ethical committees of the Helsinki and Uusimaa Hospital District approved the study protocol.

\section{Maternal depressive symptoms}

The Center for Epidemiological Studies Depression Scale (CES-D)(Radloff, 1977) was completed by the women biweekly (every two weeks) up to 14 times throughout pregnancy between 12-13 and 38-39 gestational weeks/delivery and twice up to 12 months after pregnancy. The CES-D comprises 20 questions, rated from none(0) to all(3) of the time on depressive symptoms during the past week. A score of $\geq 16$ 
indicates risk for clinical depression. The CES-D yields four subscales on depressed affect, somatic symptoms, interpersonal problems and a reverse-scored scale on well-being. We also tested whether any associations of antenatal depressive symptoms with child neurodevelopment were specific to somatic symptoms that may be a consequence of pregnancy rather than reflect depression, or if the other subscales also showed significant associations.

In the early childhood follow-up, the women completed the Beck Depression Inventory-II (BDI-II)(Beck, Steer, \& Brown, 1996), which comprises 21 items assessing depressive symptoms during the previous fortnight. Each item contains four statements, rated from 0 to 3, reflecting increasing symptom severity. A score of $\geq 14$ indicates at least mild depression.

Both depression scales have good psychometric properties(Beck et al., 1996; Radloff, 1977; Vilagut, Forero, Barbaglia, \& Alonso, 2016). The CES-D has been validated in pregnant populations(Maloni, Park, Anthony, \& Musil, 2005; Natamba et al., 2014). In our sample, the Cronbach's alphas of the CES-D during and after pregnancy varied from 0.88 to 0.92 . For the BDI-II, it was 0.90 .

\section{Child developmental milestones}

The ASQ Third edition (translated into Finnish, back-translated and publisher approved) is a reliable and valid tool with high sensitivity and specificity for screening children requiring further developmental assessment(Squires \& Bricker, 2009; Squires, Bricker, \& Potter, 1997). It includes separate questionnaires for 21 age groups between 2 and 60 months of age. Each age-specific questionnaire includes 30 ageappropriate items measuring communication, gross motor, fine motor, problem solving and personal/social (solitary social play and play with toys and other children) skills (Squires \& Bricker, 2009).

Each of the five domains domain comprises six questions with response 'yes' (scored 10) indicating the child can master the skill, 'sometimes' (scored 5) if the skill is emerging/occasional, and 'not yet' (scored 0 ) if the child cannot perform the skill. Subscale scores range from 0 to 60 , with the highest value indicating mastering all the skills in the domain. The Finnish version of the ASQ has not yet been 
normed. Hence, we used the raw ASQ total score, the mean of the subscale scores, as our primary outcome and the five raw subscale scores as secondary outcomes.

\section{Covariates}

These included maternal age at childbirth (years), occupation (entrepreneur/senior clerical/lower clerical, manual worker, student, retired or other) parity (primiparous/multiparous), maternal smoking during pregnancy (did not smoke/quit during first trimester/smoked throughout), type 1 diabetes (yes/no), chronic hypertension (yes/no), early pregnancy body mass index (BMI) (normal weight (BMI<25 $\mathrm{kg} / \mathrm{m}^{2}$ )/overweight BMI=25-29 kg/m²)/obesity $\left.\left(\mathrm{BMI} \geq 30 \mathrm{~kg} / \mathrm{m}^{2}\right)\right]$, hypertensive pregnancy disorders (preeclampsia/gestational hypertension; yes/no), and gestational diabetes (yes/no), family structure (cohabiting/married vs. living alone) child's sex, gestational length (weeks), and birthweight (grams), with data extracted from hospital records and/or MBR; maternal history of physician-diagnosed depression before pregnancy (yes/no), psychotropic medication (antidepressants/other [barbiturates, sedatives, antipsychotics, mood stabilizers]/no) and alcohol use during pregnancy (yes/no) and education level (basic/secondary vs. tertiary) were self-reported in early pregnancy, on average at 13.8 weeks (SD=4.0 weeks) of gestation. Child's age at follow-up (years) was calculated from the ASQ completion date and birth date. Participants with missing values in categorical covariates were dummy-coded to their own category.

\section{Statistical analyses}

Associations between maternal depressive symptoms during pregnancy (trimester-weighted-mean across 14 measurements) and up to 12 months after pregnancy (mean of two measurements) and in early childhood and child's ASQ total score were tested using Tobit and logistic regressions. Tobit regression accounts for the ceiling effect resulting from the ASQ not distinguishing between children mastering all agespecific skills. Depressive symptoms were treated in these analyses both as continuous [square-root 
transformed and standardized ( $m e a n=0 ; S D=1$ ) and dichotomized at cutoffs indicating clinically relevant symptoms. ASQ total score was also used as a continuous variable and dichotomized at -1SD based on ranknormalized values according to Blom's formula, indicating mild developmental delay.

We then tested associations between maternal depressive symptoms during each period with child ASQ subscale scores using ordinal logistic regressions, because each ASQ item is measured ordinally yielding subscale scores ranging from 0 to 60.

With Tobit and/or logistic regressions, we tested (1) if maternal depressive symptoms during pregnancy showed gestation-week-specific effects on child ASQ total score, (2) if CES-D subscale scores during pregnancy were associated with children's total ASQ score, and (3) if child's ASQ total score decreased and odds for scoring below -1SD in ASQ total score increased according to the number of times the mother reported clinically relevant depressive symptoms across pregnancy trimesters $(0 / 1 / 2-3)$.

With latent profile analyses (LPA), we identified time-varying trajectories in maternal biweekly-assessed depressive symptoms during pregnancy and tested with Tobit and logistic regressions if ASQ total score and the proportion of children with ASQ total score below -1SD differed between the groups identified by LPA.

Next, we tested with Tobit regression if child's ASQ total score differed between the groups of women who during pregnancy, up to 12 months after pregnancy and in early childhood did not report clinically relevant depressive symptoms (referent) and who reported clinically relevant symptoms at any time point.

For all these analyses, we present unstandardized regression coefficients (B) and/or odds ratios $(\mathrm{OR})$ and $95 \%$ confidence interval $(95 \% \mathrm{Cl})$ from models adjusted for child's age and sex, and further for maternal age, parity, education, occupation, history of depression before pregnancy, psychotropic medication use, alcohol use and smoking during pregnancy, early pregnancy BMI and hypertensive and diabetic pre-pregnancy and pregnancy disorders, family structure, gestation length, and infant's birthweight adjusted for sex and gestation length. 
Corresponding Author: Marius Lahti-Pulkkinen. 10

Finally, with Tobit regression models, we examined whether the effects of continuously treated maternal depressive symptoms during pregnancy, postpartum or in early childhood were independent of each other by controlling for the effects of depressive symptoms at either of the other time points, along with child sex and age at follow-up. 


\section{RESULTS}

Supplemental Table 2 shows the sample characteristics, Supplemental Table 3 the associations of the covariates with maternal depressive symptoms and Supplemental Table 4 with child developmental milestones. Maternal biweekly depressive symptoms during pregnancy were highly correlated $(r=0.50-$ $0.80 ;$ all $p<0.001)$. Trimester-weighted mean depressive symptoms during pregnancy correlated also significantly with mean depressive symptoms up to 12 months after pregnancy $(r=0.66 ; p<0.001)$ and in early childhood $(r=0.45 ; p<0.001)$.

Table 1 shows that higher mean maternal depressive symptoms during pregnancy and symptoms that were above the clinical cutoff associated with lower ASQ total score and higher odds of scoring below the -1SD cutoff on the total ASQ scale across both adjustment models. Higher maternal depressive symptoms during pregnancy also associated with lower ASQ subscale scores on gross and fine motor, communication, problem solving and personal/social skills across adjustment models (Supplemental Table 5). Furthermore, all maternal CES-D subscale scores during pregnancy, higher depressed affect, somatic symptoms and interpersonal problems and lower well-being, associated with significantly lower child total ASQ scores (Supplemental Table 6).

The LPA revealed four groups demonstrating highly stable antenatal depressive symptom levels (Akaike Information Criterion=177252.50; $p=0.05$ ) as the best descriptor of depressive symptoms across pregnancy (Figure 1). The two groups with lower depressive symptoms did not have clinically relevant symptomatology, while the two groups with higher depressive symptoms had subthreshold levels and levels indicating clinically relevant symptomatology. Figure 1 also shows that children whose mothers had consistently clinically relevant depressive symptoms during pregnancy had the lowest ASQ total scores and the highest proportion and odds of scoring below -1SD on the ASQ total scale. Supplemental Table 7, which shows associations between biweekly depressive symptoms scores during pregnancy and child ASQ total score, further emphasizes that the associations were independent of covariates and not gestationweek specific. Yet, child ASQ total scores decreased and the proportion and odds for having ASQ total 
Corresponding Author: Marius Lahti-Pulkkinen. 12

scores below -1SD increased linearly according to the number of pregnancy trimesters $(0 / 1 / 2-3)$ maternal depressive symptoms were above the clinical cutoff score (Figure 2).

Table 1 shows that higher maternal depressive symptoms up to 12 months after pregnancy and in early childhood and symptoms above the clinical cutoff during these time points associated with lower ASQ total score and higher odds of scoring below the -1SD cutoff on the total ASQ in both models. Higher maternal depressive symptoms up to 12 months after pregnancy and in early childhood also significantly associated with lower scores on the ASQ gross and fine motor, communication, problem solving and personal/social skills subscales (Supplemental Table 8).

Table 2 shows that of the 2,136 women with depressive symptoms data at all three time points, 437(20.5\%) scored above the clinical cutoff during pregnancy, 389(18.2\%) up to 12 months after pregnancy, and $178(8.3 \%)$ in early childhood. Compared to the children of women who did not report clinically relevant depressive symptoms at any time point, child ASQ total scores were significantly lower for the following three groups: women with depressive symptom scores above the clinical cutoff only during pregnancy, both during pregnancy and up to 12 months after pregnancy, and at all three time points (Table 2).

Supplemental Table 9 shows that when treated as continuous scores, maternal depressive symptoms during pregnancy, postpartum and in early childhood each predicted lower child developmental milestone scores independently of maternal depressive symptoms at both of the other time points. 


\section{DISCUSSION}

Higher maternal depressive symptoms during and after pregnancy associated with lower developmental milestones total score and subscale scores in fine and gross motor, communication, problem solving and personal/social skills in 1.9 to 5.7-year-old children. The pregnancy effects were independent of gestation week: children of mothers with consistently clinically relevant depressive symptoms during pregnancy had the lowest developmental milestones scores. Correspondingly, children whose mothers reported clinically relevant symptoms at two or three pregnancy trimesters had more neurodevelopmental disadvantage than children whose mothers reported clinically relevant symptoms during one or zero trimesters. Our findings were independent of important covariates that have been associated with depressive symptoms and/or poorer neurodevelopment either in this study or previously, including maternal history of physiciandiagnosed depression, early pregnancy BMI, pregnancy disorders, psychotropic medication and substance use during pregnancy, age, parity, education, occupation, family structure, child's sex, age, gestation length and birth weight, and of somatic changes accompanying pregnancy included in depressive symptoms.

Our findings verify previous findings that maternal depressive symptoms measured once or twice during pregnancy carry adverse neurodevelopmental consequences on children(Van den Bergh et al., 2017). Yet, our findings add significantly to this literature by showing that child neurodevelopmental performance worsened according to the chronicity and severity of maternal depressive symptoms during pregnancy.

Apart from antenatal depression, also higher maternal depressive symptoms up to 12 months after pregnancy and in early childhood associated with lower developmental milestones total score and lower scores across all neurodevelopmental domains in children. Maternal depressive symptoms after pregnancy clearly influenced child neurodevelopment. The after pregnancy effects were additive to those during pregnancy: children whose mothers had chronically elevated depressive symptoms during and after pregnancy had the poorest developmental milestones scores. Compared to children whose mothers never had clinically significant depressive symptoms, neurodevelopmental scores were significantly worse for 
Corresponding Author: Marius Lahti-Pulkkinen. 14

children whose mothers had clinically relevant depressive symptoms either only during pregnancy, both during and up to 12 months after pregnancy, or across all three developmental stages. Children whose mothers had clinically relevant depressive symptoms only after pregnancy trended to fare worse but did not differ systematically from children of mothers not reporting clinically relevant depressive symptoms at any developmental stage. Only two previous studies have tested similar accumulation of depressive symptoms during and after pregnancy(Evans et al., 2012; van der Waerden et al., 2017). Our findings agree with theirs by showing poorest neurodevelopment in children whose mothers were chronically depressed during and after pregnancy. Our findings with categorically defined depression also confirm their conclusion that "the postnatal period is not a sensitive one for the effect of maternal depression on child cognitive development"(Evans et al., 2012). Nevertheless, our findings partially contrast one of these studies(van der Waerden et al., 2017) but correspond with the other (Evans et al., 2012) by showing that high maternal depressive symptoms occurring specifically during pregnancy affect child neurodevelopment. Furthermore, when treated continuously, maternal depressive symptoms during pregnancy, postpartum and in early childhood each predicted poorer child neurodevelopment independently of each other. These findings confirm that the effects of maternal depressive symptoms during pregnancy on child neurodevelopment were specific to the prenatal period but, in contrast to the above-mentioned findings, also suggest specific effects of maternal depressive symptoms postpartum and in early childhood. However, the high stability of depressive symptoms, as shown by their high intercorrelations across time points, makes it difficult to distinguish antenatal, postpartum and early childhood depression effects and may have led to multicollinearity problems in the analyses. Hence, the independence of these effects must be interpreted with caution. Yet, we also show that the additive effects of depressive symptoms during and after pregnancy follow a linear pattern and the effects of maternal depression during and after pregnancy are independent of important covariates, including cardiometabolic pregnancy disorders which most previous studies did not account for.

Our findings emphasize the role of maternal depression during pregnancy on child neurodevelopment. This supports the DOHaD-hypothesis, which suggests that prenatal exposure to 
environmental adversity such as maternal depression may "program" child neurodevelopment(Barker, 2007), possibly through its effects on brain development(Van den Bergh et al., 2017). Furthermore, maternal depressive symptoms during pregnancy are also associated with maternal biomarkers with offspring neurodevelopmental relevance, including polyunsaturated fatty acids(P.Y. Lin, Chang, Chong, Chen, \& Su, 2017), proinflammatory cytokines(Christian, Franco, Glaser, \& lams, 2009), thyroid hormones(Bunevicius et al., 2009), cortisol(Seth, Lewis, \& Galbally, 2016), and placental morphology(LahtiPulkkinen et al., 2018), and with elevated placental corticotrophin releasing hormone(Moog et al., 2016), and placental mRNA gene expression levels of glucocorticoid (NR3C1) and mineralocorticoid (NR3C2) receptors(Reynolds et al., 2015; Räikkönen et al., 2015). These findings suggest that the mechanisms are complex and may include structural and functional alterations at the materno-placento-fetal unit. It has also been suggested that maternal depression during pregnancy fails to prime mother-child responsiveness(Pearson, Cooper, Penton-Voak, Lightman, \& Evans, 2010), and that the main mechanism linking maternal depression after pregnancy and child neurodevelopment is the sub-optimally developing mother-child attachment(Carter et al., 2001).

Strengths of our study include the prospective design, large and well-characterized sample, repeated depressive symptoms measurements throughout and after pregnancy, and a well-validated measure of child developmental milestones. Yet, nearly half of the cohort was lost to follow-up, and the ethnic homogeneity of our sample and use of single informant and questionnaire on child neurodevelopment limit the generalizability of our findings. While the attrition level was typical to longitudinal studies, it was not independent of maternal or child characteristics and maternal depressive symptoms were higher among non-participants. Hence, a selection bias in participation may have influenced our findings. Nevertheless, the prevalence of maternal antenatal antidepressant use in our sample corresponded to the general Finnish population(Malm et al., 2016), supporting the representativeness of our sample. On the other hand, while we focused on the effects of maternal depressive symptoms on child neurodevelopment, depression is highly comorbid with anxiety also in the perinatal period(Falah-Hassani, Shiri, \& Dennis, 2017), and maternal antenatal anxiety has also been shown 
Corresponding Author: Marius Lahti-Pulkkinen. 16

to affect child neurodevelopment(Buss, Davis, Hobel, \& Sandman, 2011; O’Donnell et al., 2017; Van den Bergh et al., 2017). Hence, further studies disentangling the unique effects of maternal depression and anxiety on child neurodevelopment are warranted. A further limitation is that we had no paternal antenatal depressive symptoms data. Further studies assessing also paternal depressive symptom trajectories and using multiple informants and/or neuropsychological tests on child neurodevelopment are needed.

Furthermore, we used cutoff scores of depressive symptoms in many analyses. Cutoff scores are sensitive to the population studied and also other cutoffs have been suggested for the CES-D(Vilagut et al., 2016). However, our findings were similar whether we used categorical or continuous depressive symptom assessments.

Clinically, our findings implicate that intervention in early pregnancy may benefit both mothers and children: early treatment may prevent the accumulative cascade of depressive symptoms from pregnancy onwards, which may all possibly reflect positively on child neurodevelopment. 


\section{CONCLUSION}

Maternal depressive symptoms during and up to 12 months after pregnancy and in early childhood associate significantly with lower child developmental milestones scores. Our longitudinal study suggests that the effects of depressive symptoms during and after pregnancy on child neurodevelopment are additive: children of women with the most chronic and severe depressive symptoms during and after pregnancy have the most neurodevelopmental disadvantages. 
Corresponding Author: Marius Lahti-Pulkkinen. 18

\section{ACKNOWLEDGEMENTS}

The PREDO study is funded by the Academy of Finland, EraNetNeuron, EVO, University of Helsinki Research Funds, and the Signe and Ane Gyllenberg, Emil Aaltonen, Jane and Aatos Erkko, Novo Nordisk, Päivikki and Sakari Sohlberg, and Sigrid Juselius Foundations, the Finnish Medical Foundation, the British Heart Foundation and the European Commission Horizon 2020 Award SC1-2016-RTD-733280 RECAP. 


\section{REFERENCES}

Barker, D. J. P. (2007). The origins of the developmental origins theory. Journal of Internal Medicine, 261(5), 412-417. http://doi.org/10.1111/j.1365-2796.2007.01809.x

Beck, A., Steer, R., \& Brown, G. (1996). BDI-II, Beck depression inventory: manual. ... for Beck Depression Inventory-II. Psychological Corporation.

Bunevicius, R., Kusminskas, L., Mickuviene, N., Bunevicius, A., Pedersen, C. a, \& Pop, V. J. M. (2009). Depressive disorder and thyroid axis functioning during pregnancy. The World Journal of Biological Psychiatry : The Official Journal of the World Federation of Societies of Biological Psychiatry, 10(4), 324-329. http://doi.org/10.3109/15622970903144038

Buss, C., Davis, E., Hobel, C., \& Sandman, C. (2011). Maternal pregnancy-specific anxiety is associated with child executive function at 6-9 years age. Stress, 14(6), 665-76.

http://doi.org/10.3109/10253890.2011.623250

Carter, S., Garrity-Rokous, F. E., Chazan-Cohen, R., Little, C., \& Briggs-Gowan, M. J. (2001). Maternal depression and comorbidity: predicting early parenting, attachment security, and toddler socialemotional problems and competencies. Journal of the American Academy of Child and Adolescent Psychiatry, 40(1), 18-26. http://doi.org/10.1097/00004583-200101000-00012

Christian, L. M., Franco, A., Glaser, R., \& lams, J. D. (2009). Depressive symptoms are associated with elevated serum proinflammatory cytokines among pregnant women. Brain, Behavior, and Immunity, 23(6), 750-754. http://doi.org/10.1016/j.bbi.2009.02.012

Chung, T. K., Lau, T. K., Yip, a S., Chiu, H. F., \& Lee, D. T. (2001). Antepartum depressive symptomatology is associated with adverse obstetric and neonatal outcomes. Psychosomatic Medicine, 63(5), 830-834. http://doi.org/10.1097/00006842-200109000-00017

DiPietro, J. A., Novak, M. F. S. X., Costigan, K. A., Atella, L. D., \& Reusing, S. P. (2006). Maternal Psychological Distress During Pregnancy in Relation to Child Development at Age Two. Child Development, 77(3), 
572-587. http://doi.org/10.1111/j.1467-8624.2006.00891.x

El Marroun, H., White, T. J., Fernandez, G., Jaddoe, V. W., Verhulst, F. C., Stricker, B. H., \& Tiemeier, H. (2016). Prenatal exposure to selective serotonin reuptake inhibitors and non-verbal cognitive functioning in childhood. Journal of Psychopharmacology (Oxford, England), 269881116665335. http://doi.org/10.1177/0269881116665335

Evans, J., Melotti, R., Heron, J., Ramchandani, P., Wiles, N., Murray, L., \& Stein, A. (2012). The timing of maternal depressive symptoms and child cognitive development: A longitudinal study. Journal of Child Psychology and Psychiatry and Allied Disciplines, 53(6), 632-640. http://doi.org/10.1111/j.14697610.2011.02513.x

Falah-Hassani, K., Shiri, R., \& Dennis, C.-L. (2017). The prevalence of antenatal and postnatal co-morbid anxiety and depression: a meta-analysis. Psychological Medicine, 47, 2041-2053. http://doi.org/10.1017/S0033291717000617

Gerardin, P., Wendland, J., Bodeau, N., Galin, A., Bialobos, S., Tordjman, S., ... Cohen, D. (2011). Depression during pregnancy: Is the developmental impact earlier in boys? A prospective case-control study. Journal of Clinical Psychiatry, 72(3), 378-387. http://doi.org/10.4088/JCP.09m05724blu

Girchenko, P., Lahti, M., Tuovinen, S., Savolainen, K., Lahti, J., Binder, E. B., ... Räikkönen, K. (2017). Cohort Profile: Prediction and prevention of preeclampsia and intrauterine growth restriction (PREDO) study. International Journal of Epidemiology, 46(5), 1380-1381g. http://doi.org/https://doi.org/10.1093/ije/dyw154

Grace, S. L., Evindar, A., \& Stewart, D. E. (2003). The effect of postpartum depression on child cognitive development and behavior: A review and critical analysis of the literature. Archives of Women's Mental Health. http://doi.org/10.1007/s00737-003-0024-6

Grigoriadis, S., VonderPorten, E. H., Mamisashvili, L., Tomlinson, G., Dennis, C.-L., Koren, G., ... Ross, L. E. (2013). The impact of maternal depression during pregnancy on perinatal outcomes: a systematic 
review and meta-analysis. The Journal of Clinical Psychiatry, 74(4), e321-41.

http://doi.org/10.4088/JCP.12r07968

Halligan, S. L., Murray, L., Martins, C., \& Cooper, P. J. (2007). Maternal depression and psychiatric outcomes in adolescent offspring: A 13-year longitudinal study. Journal of Affective Disorders, 97(1-3), 145-154. http://doi.org/10.1016/j.jad.2006.06.010

Hay, D. F., Pawlby, S., Waters, C. S., \& Sharp, D. (2008). Antepartum and postpartum exposure to maternal depression: Different effects on different adolescent outcomes. Journal of Child Psychology and Psychiatry and Allied Disciplines, 49(10), 1079-1088. http://doi.org/10.1111/j.14697610.2008.01959.x

Heron, J., O'Connor G., T., Evans, J., Golding, J., \& Glover, V. (2004). The course of anxiety and depression through pregnancy and the postpartum in a community sample. Journal of Affective Disorders, 80(1), $65-73$.

Ibanez, G., Bernard, J. Y., Rondet, C., Peyre, H., Forhan, A., Kaminski, M., ... Group, E. M.-C. C. S. (2015). Effects of Antenatal Maternal Depression and Anxiety on Children's Early Cognitive Development: A Prospective Cohort Study. PLoS One, 10(8), e0135849. http://doi.org/10.1371/journal.pone.0135849

Jarde, A., Morais, M., Kingston, D., Giallo, R., MacQueen, G. M., Giglia, L., ... McDonald, S. D. (2016). Neonatal Outcomes in Women With Untreated Antenatal Depression Compared With Women Without Depression: A Systematic Review and Meta-analysis. JAMA Psychiatry, 73(8), 826-37. http://doi.org/10.1001/jamapsychiatry.2016.0934

Koutra, K., Roumeliotaki, T., Kyriklaki, A., Kampouri, M., Sarri, K., Vassilaki, M., ... Chatzi, L. (2017). Maternal depression and personality traits in association with child neuropsychological and behavioral development in preschool years: Mother-child cohort (Rhea Study) in Crete, Greece. Journal of Affective Disorders, 217(February), 89-98. http://doi.org/10.1016/j.jad.2017.04.002

Kurstjens, S., \& Wolke, D. (2001). Effects of maternal depression on cognitive development of children over 
the first 7 years of life. Journal of Child Psychology and Psychiatry, and Allied Disciplines, 42(5), 623636. http://doi.org/10.1111/1469-7610.00758

Lahti-Pulkkinen, M., Cudmore, M. J., Haeussner, E., Schmitz, C., Pesonen, A.-K., Hämäläinen, E., ... Räikkönen, K. (2018). Placental Morphology Is Associated with Maternal Depressive Symptoms during Pregnancy and Toddler Psychiatric Problems. Scientific Reports, 8(1), 791. http://doi.org/10.1038/s41598-017-19133-9

Lahti, M., Savolainen, K., Tuovinen, S., Pesonen, A.-K., Lahti, J., Heinonen, K., ... Räikkönen, K. (2017). Maternal Depressive Symptoms During and After Pregnancy and Psychiatric Problems in Children. Journal of the American Academy of Child \& Adolescent Psychiatry, 56(1), 30-39.e7. http://doi.org/10.1016/j.jaac.2016.10.007

Lebel, C., Walton, M., Letourneau, N., Giesbrecht, G. F., Kaplan, B. J., \& Dewey, D. (2016). Prepartum and Postpartum Maternal Depressive Symptoms Are Related to Children's Brain Structure in Preschool. Biological Psychiatry, 80(11), 859-868. http://doi.org/10.1016/j.biopsych.2015.12.004

Lin, P. Y., Chang, C. H., Chong, M. F., Chen, H., \& Su, K. P. (2017). Polyunsaturated Fatty Acids in Perinatal Depression: A Systematic Review and Meta-analysis. Biological Psychiatry. http://doi.org/10.1016/j.biopsych.2017.02.1182

Lin, Y., Xu, J., Huang, J., Jia, Y., Zhang, J., Yan, C., \& Zhang, J. (2017). Effects of prenatal and postnatal maternal emotional stress on toddlers' cognitive and temperamental development. Journal of Affective Disorders, 207, 9-17. http://doi.org/10.1016/j.jad.2016.09.010

Malm, H., Brown, A. S., Gissler, M., Gyllenberg, D., Hinkka-Yli-Salomäki, S., McKeague, I. W., ... Sourander, A. (2016). Gestational Exposure to Selective Serotonin Reuptake Inhibitors and Offspring Psychiatric Disorders: A National Register-Based Study. Journal of the American Academy of Child and Adolescent Psychiatry, 55(5), 359-366. http://doi.org/10.1016/j.jaac.2016.02.013

Maloni, J. A., Park, S., Anthony, M. K., \& Musil, C. M. (2005). Measurement of antepartum depressive 
symptoms during high-risk pregnancy. Research in Nursing and Health, 28(1), 16-26.

http://doi.org/10.1002/nur.20051

Misri, S., \& Kendrick, K. (2008). Perinatal Depression , Fetal Bonding, and Mother-Child Attachment: A Review of the Literature. Current Pediatric Reviews, 4(2), 66-70. http://doi.org/10.2174/157339608784462043

Molyneaux, E., Poston, L., Ashurst-williams, S., \& Howard, L. M. (2014). Obesity and mental disorders during pregnancy and postpartum: a systematic review and meta-analysis. Obstetrics and Gynecology, 123(4), 857-867. http://doi.org/10.1097/AOG.0000000000000170.Obesity

Moog, N. K., Buss, C., Entringer, S., Shahbaba, B., Gillen, D. L., Hobel, C. J., \& Wadhwa, P. D. (2016). Maternal Exposure to Childhood Trauma Is Associated during Pregnancy with Placental-Fetal Stress Physiology. Biological Psychiatry, 79(10), 831-839. http://doi.org/10.1016/j.biopsych.2015.08.032

Natamba, B. K., Achan, J., Arbach, A., Oyok, T. O., Ghosh, S., Mehta, S., ... Young, S. L. (2014). Reliability and validity of the center for epidemiologic studies-depression scale in screening for depression among HIV-infected and -uninfected pregnant women attending antenatal services in northern Uganda: a cross-sectional study. BMC Psychiatry, 14(1), 303. http://doi.org/10.1186/s12888-014-0303-y

Nulman, I., Koren, G., Rovet, J., Barrera, M., Pulver, A., Streiner, D., \& Feldman, B. (2012). Neurodevelopment of children following prenatal exposure to venlafaxine, selective serotonin reuptake inhibitors, or untreated maternal depression. American Journal of Psychiatry, 169(11), 11651174 10p. http://doi.org/10.1176/appi.ajp.2012.11111721

O’Donnell, K. J., Glover, V., Lahti, J., Lahti, M., Edgar, R. D., Räikkönen, K., \& O’Connor, T. G. (2017). Maternal prenatal anxiety and child COMT genotype predict working memory and symptoms of ADHD. PLOS ONE, 12(6). http://doi.org/10.1371/journal.pone.0177506

Pearson, R. M., Cooper, R. M., Penton-Voak, I. S., Lightman, S. L., \& Evans, J. (2010). Depressive symptoms in early pregnancy disrupt attentional processing of infant emotion. Psychological Medicine, 40(4), 
621-31. http://doi.org/10.1017/S0033291709990961

Pesonen, A. K., Lahti, M., Kuusinen, T., Tuovinen, S., Villa, P., Hämäläinen, E., ... Räikkönen, K. (2016). Maternal prenatal positive affect, depressive and anxiety symptoms and birth outcomes: The PREDO study. PLoS ONE, 11(2), 1-13. http://doi.org/10.1371/journal.pone.0150058

Plamondon, A., Akbari, E., Atkinson, L., Steiner, M., Meaney, M. J., \& Fleming, A. S. (2015). Spatial working memory and attention skills are predicted by maternal stress during pregnancy. Early Human Development, 91(1), 23-29. http://doi.org/10.1016/j.earlhumdev.2014.11.004

Qiu, A., Anh, T. T., Li, Y., Chen, H., Rifkin-Graboi, A., Broekman, B. F. P., ... Meaney, M. J. (2015). Prenatal maternal depression alters amygdala functional connectivity in 6-month-old infants. Translational Psychiatry, 5(2), e508. http://doi.org/10.1038/tp.2015.3

Radloff, L. S. (1977). The CES-D Scale. Applied Psychological Measurement, 1(3), 385-401. http://doi.org/10.1177/014662167700100306

Reynolds, R. M., Pesonen, A.-K., O’Reilly, J. R., Tuovinen, S., Lahti, M., Kajantie, E., ... Räikkönen, K. (2015). Maternal depressive symptoms throughout pregnancy are associated with increased placental glucocorticoid sensitivity. Psychological Medicine, 45, 2023-2030.

http://doi.org/10.1017/S003329171400316X

Rifkin-Graboi, A., Bai, J., Chen, H., Hameed, W. B. R., Sim, L. W., Tint, M. T., ... Qiu, A. (2013). Prenatal maternal depression associates with microstructure of right amygdala in neonates at birth. Biological Psychiatry, 74(11), 937-944. http://doi.org/10.1016/j.biopsych.2013.06.019

Räikkönen, K., Pesonen, A.-K., O’Reilly, J. R., Tuovinen, S., Lahti, M., Kajantie, E., ... Reynolds, R. M. (2015). Maternal depressive symptoms during pregnancy, placental expression of genes regulating glucocorticoid and serotonin function and infant regulatory behaviors. Psychological Medicine, 45(15), 3217-3226. http://doi.org/10.1017/S003329171500121X

Sandman, C. A., Buss, C., Head, K., \& Davis, E. P. (2015). Fetal exposure to maternal depressive symptoms is 
associated with cortical thickness in late childhood. Biological Psychiatry, 77(4), 324-34. http://doi.org/10.1016/j.biopsych.2014.06.025

Schechter, J. C., Brennan, P. A., Smith, A. K., Stowe, Z. N., Newport, D. J., \& Johnson, K. C. (2016). Maternal Prenatal Psychological Distress and Preschool Cognitive Functioning: the Protective Role of Positive Parental Engagement. Journal of Abnormal Child Psychology, 1-12. http://doi.org/10.1007/s10802016-0161-9

Scheinost, D., Sinha, R., Cross, S. N., Kwon, S. H., Sze, G., Constable, R. T., \& Ment, L. R. (2017). Does prenatal stress alter the developing connectome? Pediatric Research, 81(1-2), 214-226. http://doi.org/10.1038/pr.2016.197

Seth, S., Lewis, A. J., \& Galbally, M. (2016). Perinatal maternal depression and cortisol function in pregnancy and the postpartum period: a systematic literature review. BMC Pregnancy and Childbirth, 16(1), 124. http://doi.org/10.1186/s12884-016-0915-y

Soe, N. N., Wen, D. J., Poh, J. S., Chong, Y. S., Broekman, B. F., Chen, H., ... Qiu, A. (2017). Perinatal maternal depressive symptoms alter amygdala functional connectivity in girls. Human Brain Mapping, (October), 1-11. http://doi.org/10.1002/hbm.23873

Squires, J., \& Bricker, D. (2009). Ages \& Stages Questionnaires. Third Edition. (ASQ-3): A Parent-Completed Child-Monitoring System. Paul Brookes Publishing Company.

Squires, J., Bricker, D., \& Potter, L. (1997). Revision of a parent-completed developmental screening tool: Ages and stages questionnaires. Journal of Pediatric Psychology, 22(3), 313-328. http://doi.org/10.1093/jpepsy/22.3.313

Van den Bergh, B. R. H., van den Heuvel, M. I., Lahti, M., Braeken, M., de Rooij, S. R., Entringer, S., ... Schwab, M. (2017). Prenatal developmental origins of behavior and mental health: The influence of maternal stress in pregnancy. Neuroscience \& Biobehavioral Reviews. http://doi.org/10.1016/j.neubiorev.2017.07.003 
Corresponding Author: Marius Lahti-Pulkkinen. 26

van der Waerden, J., Bernard, J. Y., De Agostini, M., Saurel-Cubizolles, M. J., Peyre, H., Heude, B., ... Thiebaugeorges, O. (2017). Persistent maternal depressive symptoms trajectories influence children's IQ: The EDEN mother-child cohort. Depression and Anxiety, 34(2), 105-117. http://doi.org/10.1002/da.22552

Vilagut, G., Forero, C. G., Barbaglia, G., \& Alonso, J. (2016). Screening for Depression in the General Population with the Center for Epidemiologic Studies Depression (CES-D): A Systematic Review with Meta-Analysis. PloS One, 11(5), e0155431. http://doi.org/10.1371/journal.pone.0155431

Weikum, W. M., Oberlander, T. F., Hensch, T. K., \& Werker, J. F. (2012). Prenatal exposure to antidepressants and depressed maternal mood alter trajectory of infant speech perception. Proceedings of the National Academy of Sciences of the United States of America, 109 Supp/(Supplement_2), 17221-7. http://doi.org/10.1073/pnas.1121263109 
Table 1. Maternal depressive symptoms during and up to 12 months after pregnancy and in early childhood and child developmental milestones.

\begin{tabular}{|c|c|c|c|c|}
\hline \multirow[t]{3}{*}{ Maternal depressive symptoms } & \multirow{3}{*}{$\begin{array}{l}\text { Child Ages and Stages } \\
\text { Questionnaire score } \\
\text { B }(95 \% \mathrm{Cl})\end{array}$} & \multirow{2}{*}{\multicolumn{3}{|c|}{$\begin{array}{c}\text { Child Ages and Stages } \\
\text { Questionnaire score } \leq-1 S D\end{array}$}} \\
\hline & & & & \\
\hline & & $p$ & OR $(95 \% \mathrm{Cl})$ & $p$ \\
\hline \multicolumn{5}{|c|}{ Pregnancy mean CES-D score $(n=2231)$} \\
\hline Model 1 & $-0.99(-1.28 ;-0.71)$ & $<0.001$ & $1.40(1.25 ; 1.57)$ & $<0.001$ \\
\hline Model 2 & $-0.84(-1.13 ;-0.55)$ & $<0.001$ & $1.33(1.17 ; 1.50)$ & $<0.001$ \\
\hline \multicolumn{5}{|l|}{ Pregnancy mean CES-D score $\geq 16$} \\
\hline Model 1 & $-1.87(-2.56 ;-1.18)$ & $<0.001$ & $2.05(1.60 ; 2.64)$ & $<0.001$ \\
\hline Model 2 & $-1.56(-2.26 ;-0.87)$ & $<0.001$ & $1.92(1.47 ; 2.51)$ & $<0.001$ \\
\hline \multicolumn{5}{|c|}{ After pregnancy mean CES-D score $(n=2192)$} \\
\hline Model 1 & $-1.05(-1.34 ;-0.76)$ & $<0.001$ & $1.36(1.21 ; 1.53)$ & $<0.001$ \\
\hline Model 2 & $-0.89(-1.18 ;-0.60)$ & $<0.001$ & $1.29(1.14 ; 1.45)$ & $<0.001$ \\
\hline \multicolumn{5}{|c|}{ After pregnancy mean CES-D score $\geq 16$} \\
\hline Model 1 & $-2.13(-2.86 ;-1.40)$ & $<0.001$ & $1.85(1.42 ; 2.41)$ & $<0.001$ \\
\hline Model 2 & $-1.80(-2.53 ;-1.07)$ & $<0.001$ & $1.68(1.28 ; 2.21)$ & $<0.001$ \\
\hline \multicolumn{5}{|c|}{ Early childhood BDI-II score $(n=2170)$} \\
\hline Model 1 & $-0.97(-1.26 ;-0.69)$ & $<0.001$ & $1.27(1.13 ; 1.42)$ & $<0.001$ \\
\hline Model 2 & $-0.85(-1.14 ;-0.57)$ & $<0.001$ & $1.22(1.09 ; 1.38)$ & 0.001 \\
\hline \multicolumn{5}{|l|}{ Early childhood BDI-II score $\geq 14$} \\
\hline Model 1 & $-2.04(-2.92 ;-1.17)$ & $<0.001$ & $1.71(1.26 ; 2.34)$ & 0.001 \\
\hline Model 2 & $-1.77(-2.64 ;-0.90)$ & $<0.001$ & $1.61(1.17 ; 2.23)$ & 0.004 \\
\hline
\end{tabular}

CES-D=Center for Epidemiological Studies Depression Scale; BDI=Beck Depression Inventory-II; ASQ=Ages and Stages Questionnaire; SD=standard deviation; B=unstandardized regression coefficient; $\mathrm{OR}=$ odds ratio; $95 \% \mathrm{Cl}=95 \%$ confidence interval

For continuous depression scores the B:s and 95\% Cl:s from Tobit regression analyses refer to one-point change in ASQ scores per SD unit change in maternal depressive symptoms; for categorical depression variables the B:s and 95\% Cl:s refer to differences in ASQ scores of children of mothers with vs. without clinically relevant depressive symptoms 
Corresponding Author: Marius Lahti-Pulkkinen. 28

For continuous depression scores the ORs and 95\% Cls from logistic regression analyses show the odds of belonging to the group scoring $\leq-1 S D$ in ASQ per SD unit change in maternal depressive symptoms; for categorical depression variables the ORs and $95 \%$ Cls show the odds of belonging to the group scoring $\leq-$ $1 S D$ vs. >-1SD in ASQ for the children of mothers with vs. without clinically relevant depressive symptoms. Model 1 is adjusted for child's age and sex.

Model 2 further for maternal age, parity, education, occupation, history of depression, antidepressant and other psychotropic medication use, alcohol use and smoking during pregnancy, type 1 diabetes, chronic hypertension, early pregnancy body mass index, gestational diabetes, and hypertensive pregnancy disorders, family structure, gestational length, and child's birthweight for gestation. 
Corresponding Author: Marius Lahti-Pulkkinen. 29

Table 2. The pattern of maternal depressive symptom scores above and below the clinical cutoff over time and child developmental milestones ( $n=2136)$

\begin{tabular}{|c|c|c|c|c|c|c|c|c|}
\hline $\begin{array}{c}\text { During } \\
\text { pregnancy }\end{array}$ & $\begin{array}{l}\text { Up to } 12 \\
\text { months } \\
\text { after } \\
\text { pregnancy }\end{array}$ & $\begin{array}{c}\text { Early } \\
\text { childhood }\end{array}$ & $n$ & $\%$ & $\begin{array}{c}\text { Ages and } \\
\text { stages } \\
\text { questionnair } \\
\text { e score }^{\mathrm{a}}\end{array}$ & SE & $95 \% \mathrm{Cl}$ & $p^{\mathrm{b}}$ \\
\hline No & No & No & 1436 & 67.2 & 54.33 & 0.16 & $54.01-54.65$ & ref \\
\hline Yes & No & No & 168 & 7.9 & 53.18 & 0.48 & $52.25-54.11$ & $0.04^{c}$ \\
\hline No & Yes & No & 131 & 6.1 & 53.52 & 0.54 & $52.46-54.58$ & 0.15 \\
\hline No & No & Yes & 97 & 4.6 & 53.34 & 0.63 & $52.10-54.57$ & 0.17 \\
\hline \multirow[t]{2}{*}{ Yes } & Yes & No & 142 & 6.6 & & & & $<0.001$ \\
\hline & & & & & 52.06 & 0.52 & $51.05-53.08$ & d \\
\hline Yes & No & Yes & 45 & 2.1 & 53.43 & 0.92 & $51.62-55.23$ & 0.32 \\
\hline No & Yes & Yes & 34 & 1.6 & 52.36 & 1.06 & $50.28-54.44$ & 0.051 \\
\hline \multirow[t]{2}{*}{ Yes } & Yes & Yes & 82 & 3.8 & & & & $<0.001$ \\
\hline & & & & & 49.95 & 0.68 & $48.61-51.30$ & d \\
\hline
\end{tabular}

$\mathrm{SE}=$ standard error; $95 \% \mathrm{Cl}=95 \%$ confidence interval

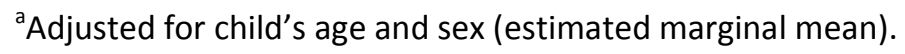

${ }^{\mathrm{b}} p$-values in Tobit regression model 1 with the group with mothers whose depressive symptom scores were never above the clinical cutoff as a referent.

${ }^{c} p$-value in Tobit regression model $2=0.07$.

${ }^{d} p$-values in Tobit regression model $2<0.001$.

For different adjustment models in the Tobit regressions, please see footnote in Table 1. 
Corresponding Author: Marius Lahti-Pulkkinen. 30

\section{FIGURE LEGENDS}

\section{Figure 1.}

Panel A: Latent profile analysis on the course of maternal depressive symptoms during pregnancy. The figure shows the mean depressive symptom scores at different gestational weeks in four groups of mothers with consistently low, subthreshold and clinically relevant depressive symptoms.

Panel B: Estimated marginal means of ASQ scores in four groups identified by latent profile analysis.

Panel C: Proportion of children with ASQ scores $\leq-1$ SD. Error bars refer to $95 \% \mathrm{Cls.}$

Numbers refer to unstandardized regression coefficients of Tobit regression analyses (Panel B) and odds ratios (Panel C) and their 95\% Cls with the group "Low1: no clinically relevant depressive symptom" as the referent and are from models adjusting for child's age and sex.

ASQ=Ages and Stages Questionnaire; CES-D=Center for Epidemiological Studies Depression Scale; $\mathrm{SD}=$ standard deviation; $95 \% \mathrm{Cl}=95 \%$ confidence interval

\section{Figure 2.}

Additive effects of maternal trimester-specific depressive symptoms during pregnancy on child developmental milestones.

Panel A: Estimated marginal means of the child Ages and Stages Questionnaire (ASQ) scores according to the number of pregnancy trimesters during which the mother had mean depressive symptoms above the clinical cutoff of $\geq 16$ are shown. Estimated marginal means are adjusted for child's age and sex.

Panel B: Percentages of children having the ASQ scores of $\leq-1 S D$ according to the number of pregnancy trimesters during which the mother had mean depressive symptoms above the clinical the clinical cutoff of $\geq 16$.

Error bars refer to $95 \%$ confidence intervals ( $95 \% \mathrm{Cls}$ ) and numbers to unstandardized regression coefficients of Tobit regression analyses (Panel A) and odds ratios (Panel B) and their $95 \%$ Cls with the group with mothers whose trimester-specific depressive symptom scores were not above the clinical cutoff at any trimester as a referent and are from models adjusting for child's age and sex. 
Table 1. Maternal depressive symptoms during and up to 12 months after pregnancy and in early childhood and child developmental milestones.

\begin{tabular}{|c|c|c|c|c|}
\hline \multirow[t]{3}{*}{ Maternal depressive symptoms } & \multirow{2}{*}{$\begin{array}{l}\text { Child Ages and Stages } \\
\text { Questionnaire score }\end{array}$} & \multicolumn{3}{|c|}{ Child Ages and Stages } \\
\hline & & \multicolumn{3}{|c|}{ Questionnaire score $\leq-1 S D$} \\
\hline & $\mathrm{B}(95 \% \mathrm{Cl})$ & $p$ & OR $(95 \% \mathrm{Cl})$ & $p$ \\
\hline \multicolumn{5}{|c|}{ Pregnancy mean CES-D score $(n=2231)$} \\
\hline Model 1 & $-0.99(-1.28 ;-0.71)$ & $<0.001$ & $1.40(1.25 ; 1.57)$ & $<0.001$ \\
\hline Model 2 & $-0.84(-1.13 ;-0.55)$ & $<0.001$ & $1.33(1.17 ; 1.50)$ & $<0.001$ \\
\hline \multicolumn{5}{|l|}{ Pregnancy mean CES-D score $\geq 16$} \\
\hline Model 1 & $-1.87(-2.56 ;-1.18)$ & $<0.001$ & $2.05(1.60 ; 2.64)$ & $<0.001$ \\
\hline Model 2 & $-1.56(-2.26 ;-0.87)$ & $<0.001$ & $1.92(1.47 ; 2.51)$ & $<0.001$ \\
\hline \multicolumn{5}{|c|}{ After pregnancy mean CES-D score $(n=2192)$} \\
\hline Model 1 & $-1.05(-1.34 ;-0.76)$ & $<0.001$ & $1.36(1.21 ; 1.53)$ & $<0.001$ \\
\hline Model 2 & $-0.89(-1.18 ;-0.60)$ & $<0.001$ & $1.29(1.14 ; 1.45)$ & $<0.001$ \\
\hline \multicolumn{5}{|c|}{ After pregnancy mean CES-D score $\geq 16$} \\
\hline Model 1 & $-2.13(-2.86 ;-1.40)$ & $<0.001$ & $1.85(1.42 ; 2.41)$ & $<0.001$ \\
\hline Model 2 & $-1.80(-2.53 ;-1.07)$ & $<0.001$ & $1.68(1.28 ; 2.21)$ & $<0.001$ \\
\hline \multicolumn{5}{|c|}{ Early childhood BDI-II score $(n=2170)$} \\
\hline Model 1 & $-0.97(-1.26 ;-0.69)$ & $<0.001$ & $1.27(1.13 ; 1.42)$ & $<0.001$ \\
\hline Model 2 & $-0.85(-1.14 ;-0.57)$ & $<0.001$ & $1.22(1.09 ; 1.38)$ & 0.001 \\
\hline \multicolumn{5}{|l|}{ Early childhood BDI-II score $\geq 14$} \\
\hline Model 1 & $-2.04(-2.92 ;-1.17)$ & $<0.001$ & $1.71(1.26 ; 2.34)$ & 0.001 \\
\hline Model 2 & $-1.77(-2.64 ;-0.90)$ & $<0.001$ & $1.61(1.17 ; 2.23)$ & 0.004 \\
\hline
\end{tabular}

CES-D=Center for Epidemiological Studies Depression Scale; BDI=Beck Depression Inventory-II; ASQ=Ages and Stages Questionnaire; SD=standard deviation; B=unstandardized regression coefficient; OR=odds ratio; $95 \% \mathrm{Cl}=95 \%$ confidence interval

For continuous depression scores the B:s and 95\% Cl:s from Tobit regression analyses refer to one-point change in ASQ scores per SD unit change in maternal depressive symptoms; for categorical depression variables the B:s and 95\% Cl:s refer to differences in ASQ scores of children of mothers with vs. without clinically relevant depressive symptoms

For continuous depression scores the ORs and 95\% Cls from logistic regression analyses show the odds of belonging to the group scoring S-1SD in ASQ per SD unit change in maternal depressive symptoms; for 
categorical depression variables the ORs and 95\% Cls show the odds of belonging to the group scoring $\leq-$ $1 S D$ vs. >-1SD in ASQ for the children of mothers with vs. without clinically relevant depressive symptoms. Model 1 is adjusted for child's age and sex.

Model 2 further for maternal age, parity, education, occupation, history of depression, antidepressant and other psychotropic medication use, alcohol use and smoking during pregnancy, type 1 diabetes, chronic hypertension, early pregnancy body mass index, gestational diabetes, and hypertensive pregnancy disorders, family structure, gestational length, and child's birthweight for gestation. 
Table 2. The pattern of maternal depressive symptom scores above and below the clinical cutoff over time and child developmental milestones ( $n=2136)$

\begin{tabular}{|c|c|c|c|c|c|c|c|c|}
\hline $\begin{array}{c}\text { During } \\
\text { pregnancy }\end{array}$ & $\begin{array}{l}\text { Up to } 12 \\
\text { months } \\
\text { after } \\
\text { pregnancy }\end{array}$ & $\begin{array}{c}\text { Early } \\
\text { childhood }\end{array}$ & $\mathrm{n}$ & $\%$ & $\begin{array}{c}\text { Ages and } \\
\text { stages } \\
\text { questionnair } \\
\text { e score }^{\mathrm{a}}\end{array}$ & SE & $95 \% \mathrm{Cl}$ & $p^{b}$ \\
\hline No & No & No & 1436 & 67.2 & 54.33 & 0.16 & 54.01-54.65 & ref \\
\hline Yes & No & No & 168 & 7.9 & 53.18 & 0.48 & $52.25-54.11$ & $0.04^{c}$ \\
\hline No & Yes & No & 131 & 6.1 & 53.52 & 0.54 & $52.46-54.58$ & 0.15 \\
\hline No & No & Yes & 97 & 4.6 & 53.34 & 0.63 & $52.10-54.57$ & 0.17 \\
\hline \multirow[t]{2}{*}{ Yes } & Yes & No & 142 & 6.6 & & & & $<0.001$ \\
\hline & & & & & 52.06 & 0.52 & $51.05-53.08$ & d \\
\hline Yes & No & Yes & 45 & 2.1 & 53.43 & 0.92 & $51.62-55.23$ & 0.32 \\
\hline No & Yes & Yes & 34 & 1.6 & 52.36 & 1.06 & $50.28-54.44$ & 0.051 \\
\hline \multirow[t]{2}{*}{ Yes } & Yes & Yes & 82 & 3.8 & & & & $<0.001$ \\
\hline & & & & & 49.95 & 0.68 & $48.61-51.30$ & $d$ \\
\hline
\end{tabular}

$\mathrm{SE}=$ standard error; $95 \% \mathrm{Cl}=95 \%$ confidence interval

${ }^{\text {a} A d j u s t e d ~ f o r ~ c h i l d ' s ~ a g e ~ a n d ~ s e x ~(e s t i m a t e d ~ m a r g i n a l ~ m e a n) . ~}$

${ }^{\mathrm{b}} p$-values in Tobit regression model 1 with the group with mothers whose depressive symptom scores were never above the clinical cutoff as a referent.

${ }^{c} p$-value in Tobit regression model $2=0.07$.

${ }^{\mathrm{d}} p$-values in Tobit regression model $2<0.001$.

For different adjustment models in the Tobit regressions, please see footnote in Table 1. 
Panel A

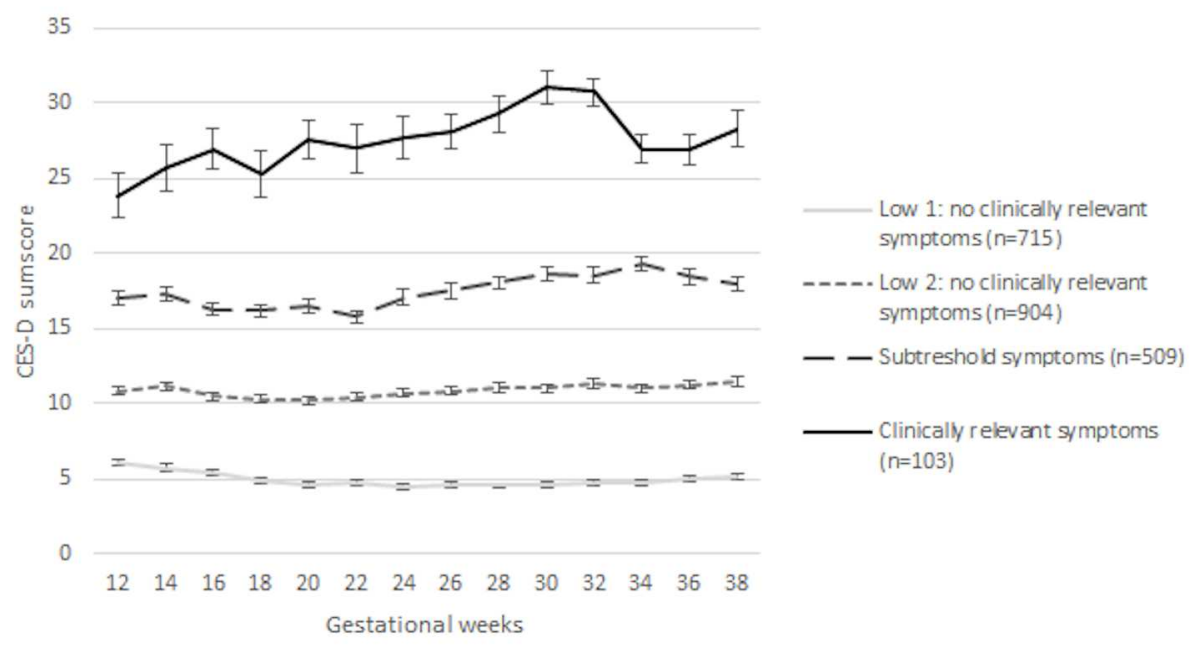

Panel B

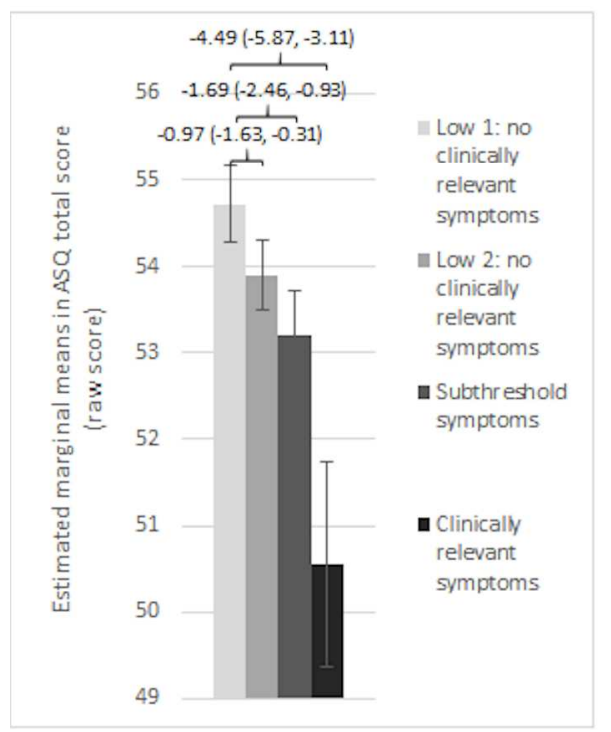

Panel C

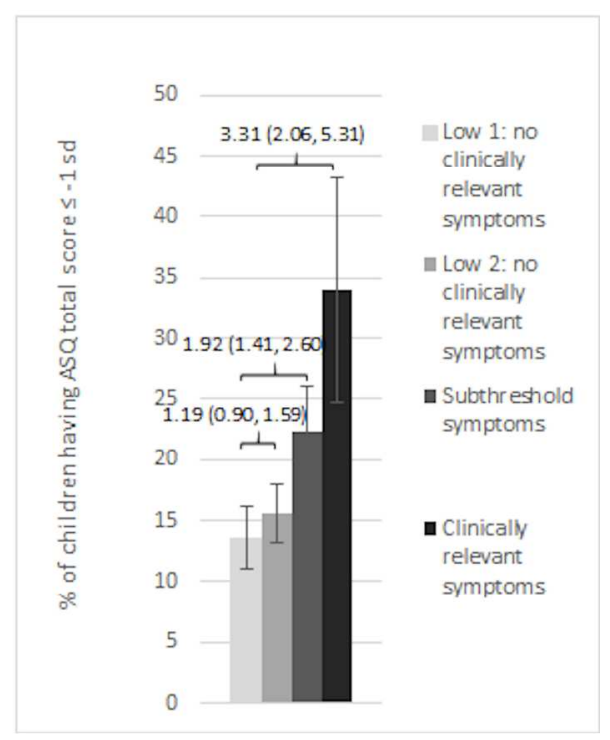

Figure 1. "Panel A: Latent profile analysis on the course of maternal depressive symptoms during pregnancy. The figure shows the mean depressive symptom scores at different gestational weeks in four groups of mothers with consistently low, subthreshold and clinically relevant depressive symptoms. "Panel B: Estimated marginal means of ASQ scores in four groups identified by latent profile analysis. "Panel C:

Proportion of children with ASQ scores $\leq-1$ SD. Error bars refer to $95 \%$ CIs. "Numbers refer to unstandardized regression coefficients of Tobit regression analyses (Panel B) and odds ratios (Panel C) and their 95\% CIs with the group "Low1: no clinically relevant depressive symptom" as the referent and are from models adjusting for child's age and sex. "ASQ=Ages and Stages Questionnaire; CES-D=Center for

Epidemiological Studies Depression Scale; SD=standard deviation; $95 \% \mathrm{CI}=95 \%$ confidence interval

$$
151 \times 191 \mathrm{~mm}(600 \times 600 \mathrm{DPI})
$$



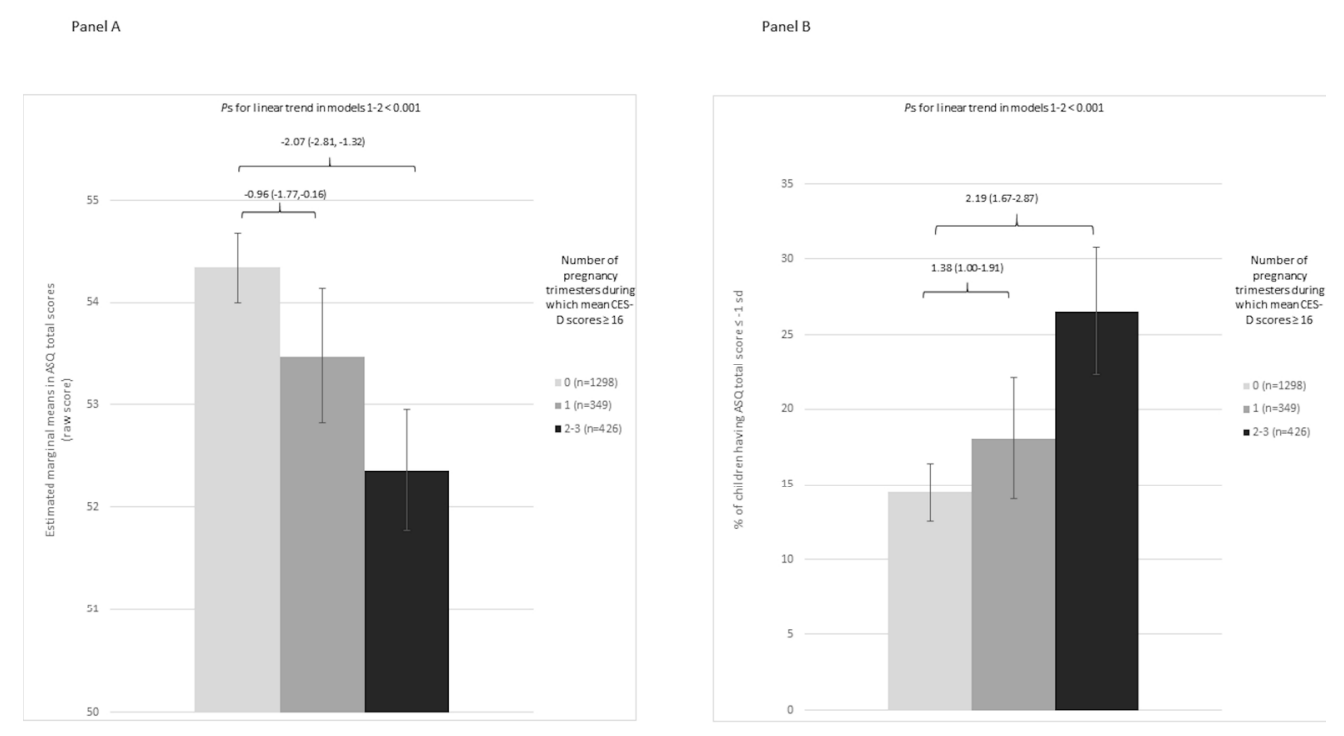

Figure 2. !! + Additive effects of maternal trimester-specific depressive symptoms during pregnancy on child developmental milestones. !! + Panel A: Estimated marginal means of the child Ages and Stages

Questionnaire (ASQ) scores according to the number of pregnancy trimesters during which the mother had mean depressive symptoms above the clinical cutoff of $\geq 16$ are shown. Estimated marginal means are adjusted for child's age and sex. !! + Panel B: Percentages of children having the ASQ scores of $\leq-1$ SD according to the number of pregnancy trimesters during which the mother had mean depressive symptoms above the clinical the clinical cutoff of $\geq 16$. ! + Error bars refer to $95 \%$ confidence intervals (95\% CIs) and numbers to unstandardized regression coefficients of Tobit regression analyses (Panel $A$ ) and odds ratios (Panel B) and their 95\% CIs with the group with mothers whose trimester-specific depressive symptom scores were not above the clinical cutoff at any trimester as a referent and are from models adjusting for child's age and sex. !! +

$95 \times 53 \mathrm{~mm}(600 \times 600 \mathrm{DPI})$ 